\title{
LAS COMPETENCIAS DOCENTES EN EL SIGLO XXI DE CARA A LA VIRTUALIDAD DE LA EDUCACIÓN CON OCASIÓN DEL COVID-19
}

\section{TEACHING COMPETENCIES IN THE 21stCENTURY FACING THE VIRTUALITY OF EDUCATION IN LIGHT OF COVID-19}

\section{Cecilia Garzón Daza ${ }^{1}$}

Colombia

\section{RESUMEN}

La virtualidad de la educación es un tema que ya tiene un recorrido amplio, pues esta comenzó con la educación a distancia hasta llegar a lo que actualmente conocemos como educación virtual; no obstante, dicho avance fue concebido para quienes por múltiples circunstancias no podían formarse por el método tradicional, permitiendo generar un espacio de gran trascendencia para

$1 \quad$ Postdoctora en Educación (Universidad Santo Tomás), Doctora en Ciencias de la educación (Universidad Pedagógica y Tecnológica de Colombia), Maestría en Administración de Empresas (Universidad de Chapultepec), Maestría en Educación (Universidad Javeriana), Contadora Pública (Universidad Antonio Nariño). Docente investigadora de la Fundación Universitaria San Mateo. Directora de calidad Universidad Católica de Colombia. https://orcid. orq/0000-0002-4173-2609

Cecilia Garzon Daza ceciliagarzon3@hotmail.com estas personas y así no perder la posibilidad de educarse.

Sin embargo, dadas las condiciones actuales del mundo y los cambios que abruptamente se introdujeron en la vida, con ocasión de la pandemia COVID - 19, la virtualidad dejó de ser una opción para convertirse en una realidad, que al parecer día a día se va a posicionar más, al punto de incluir en este proceso a los más pequeños. Esta situación exige un cambio, reacondicionamiento, reestructuración y optimización de las competencias docentes tal como se plantea en la agenda E2030: educación y habilidades para el siglo XXI. 
En este orden de ideas, el presente artículo tiene como fin evidenciar las necesidades pertinentes de las competencias docentes, que se deben afianzar para los cambios que la educación ha tenido y que el docente debe asumir sin importar el nivel de formación al que se dirija; de esta forma el país continuará respondiendo a los fines de calidad y competitividad en la aldea global del conocimiento.

Lo anterior, se estructura a partir de un diseño metodológico cualitativo con una técnica documental, mediante las cuales se busca lograr la información pertinente para fijar los parámetros que se deben seguir para estar a la altura y exigencias del mundo actual frente a lo que se está viviendo con la pandemia mundial.

\section{PALABRAS CLAVE}

competencias docentes, educación virtual, pandemia COVID - 19, calidad y competitividad.

\section{SUMMARY}

The virtuality of education is a topic that already has a long history, since it began with distance education until it reached what we currently know as virtual education. However, this advance was conceived for those who, due to multiple circumstances, could not be trained by the traditional method, allowing for the generation of a space of great importance for these people who would thus not lose the possibility of being educated.

However, given the current conditions of the world and the changes that were abruptly introduced in life following the COVID-19 pandemic, virtuality is no longer an option but a reality, which apparently day by day, is going to position itself more, to the point of including the youngest in this process. This situation demands a change, reconditioning, restructuring and optimization of teaching competencies as stated in the E2030 agenda: education and skills for the 21 st century.
In this order of ideas, the purpose of this article is to show the pertinent needs of teaching competencies, which must be strengthened for the changes that education has undergone and that teachers must respond to, regardless of the level of training to which they are directed. In this way, the country will continue to respond to the goals of quality and competitiveness in the global village of knowledge.

The above is structured from a qualitative methodological design with a documentary technique, through which it seeks to achieve the relevant information to set the parameters which must be followed to meet the demands of today's world in the face of what is being experienced with the first global pandemic.

\section{KEY WORDS}

teaching competencies, virtual education, COVID-19 pandemic, quality and competitiveness.

\section{INTRODUCCIÓN}

Las competencias docentes sin duda alguna hacen parte de la columna vertebral de la educación, pues de ellas depende la comprensión que los estudiantes hagan sobre el proceso de enseñanza - aprendizaje. Desde esta perspectiva las competencias docentes integran el sentir mismo de la educación, su razón de ser, permitiendo la articulación entre las normas, procesos, mecanismos, recursos e infraestructura con la que cuenta la educación para formar a niños, niñas y jóvenes.

Las competencias docentes entonces deben ser vistas como el día a día de la educación; toda vez, que éstas deben responder a las demandas de la formación con la inmediatez que ésta requiere; es por ello, que dichas competencias deben siempre ser vistas como algo estructural y vital para la educación. Lo anterior, teniendo en cuenta que dichas competencias se entienden 
como los recursos, habilidades, conocimiento $y$ actitudes que deben tener los docentes para atender de forma pronta y efectiva, todas las situaciones que se desprenden de su ejercicio profesional.

Las competencias no deben ser vistas de forma estática, rígida e inmodificables; por el contrario, estas deben responder al contexto, situaciones y espacios en los que el docente se desenvuelve; permitiendo desarrollar de la mejor forma posible ambientes de aprendizaje acorde a las necesidades de los estudiantes e igualmente, generar las herramientas de evaluación apropiadas para hacer el correspondiente seguimiento a las dificultades que tengan los estudiantes y, en consecuencia, apoyarlos.

En este orden de ideas se puede inferir que las competencias docentes deben responder a los cambios que la humanidad ha tenido, particularmente con el auge de la tecnología que día a día ocupa un espacio más protagónico en la vida de las personas. Así, las competencias docentes deben enfrentar los cambios que el siglo XXI introdujo en pro de fortalecer nuevas habilidades que le exigen al educador un rol más dinámico dentro de su ejercicio profesional. Rol que con ocasión de la pandemia COVID - 19 tuvo un papel importante al verse la educación volcada a la virtualidad; en donde quedó al descubierto los pros y contra de las competencias docentes en general y en particular las destrezas, actitudes y aptitudes de los docentes para enfrentar dicha situación.

Lo expresado hasta ahora lleva a formular el siguiente cuestionamiento que tiene como finalidad fijar las directrices para el desarrollo del presente artículo de reflexión: ¿reconocen los docentes la importancia de las competencias de cara a la virtualidad de la educación con ocasión del COVID - 19? La importancia del reconocimiento en este contexto resulta de gran trascendencia, pues de éste depende que el docente tenga una postura autocrítica que le va a permitir generar, no solamente los espacios de apropiación para la nueva situación, sino que le exige revisar su proceso formativo para adecuarlo a las necesidades propias de la virtualidad de la educación.

Así, se tiene como finalidad para el artículo realizar un análisis reflexivo sobre las competencias docentes en el siglo XXI de cara a la virtualidad de la educación con ocasión de la pandemia-COVD 19. Como es comprensible todavía no salimos del asombro de pasar de una vida rutinaria, habitual, común y aceptada por todos, a una vida en aislamiento en donde la tecnología se convirtió en el presente y futuro de la sociedad, exigiendo con la inmediatez que se requiere los cambios pertinentes para atender lo que habitualmente realizábamos en espacios materiales, en tiempos concretos y en contacto con nuestros estudiantes y compañeros de trabajo, virtualmente, todo quedó metido, adecuado o resumido en un computador, Tablet, celular o cualquier dispositivo que nos permita la conectividad que necesitamos para continuar con la vida cotidiana desde cualquier espacio de nuestros hogares.

El artículo se desarrolla a través de una investigación descriptiva con una técnica documental que sirve para la revisión, análisis e interpretación de los documentos que se requieran consultar, para el abordaje de lo planteado en la pregunta de investigación y el objetivo propuesto.

\section{LAS COMPETENCIAS DOCENTES ENTRE LOS PILARES DE LA TRANSFORMACIÓN Y LOS DESAFÍOS DE LA VIRTUALIDAD EDUCATIVA}

Para nadie es desconocido que la virtualidad de la educación viene haciendo presencia, desde antes de que el Siglo XXI entrará en todo su esplendor. "La virtualización es parte de la transformación digital que impulsa la revolución tecnológica, en la cual el acceso a la 
educación virtual se constituye en un derecho educativo fundamental" (Medina, 2019, pág. 215). La virtualización de la educación ha sido la innovación más significativa para los procesos formativos en el contexto global, ya que le permitió a la educación superior llegar a más estudiantes que por motivos laborales, de tiempo y ubicación no podían acceder a ésta y que en la actualidad llega a todos los niveles de educación desde la primaria hasta los estudios doctorales.

Situación que se ha forjado, gracias a que la virtualidad de la educación desde sus inicios se ha caracterizado por integrar una gran "variedad y dinamismo metodológicos, perfilándose como una panacea, aunque aún sin estrategias claras debido a los mitos y prejuicios que conlleva su tratamiento, al ofrecer ampliar las oportunidades de acceso" (Nieto, 2012, pág. 137) a quienes tienen limitada la formación por los métodos tradicionales.

La virtualidad en la educación exige no solamente el uso de "equipos, software, tecnologías inalámbrica y redes de banda ancha, sino la conceptualización de los roles, conocimientos y destrezas de los actores del proceso que permitan no sólo la reproducción, sino la comprensión de los contenidos programados en el aula virtual" (Arraiz, 2012, pág. 77). Desde esta perspectiva la virtualidad de la educación es más exigente que los métodos tradicionales, debido a que éstos perduraron por años, casi inamovibles en la manera como se educaban a los estudiantes. Los cambios obedecían a temas que modernizaban las diferentes áreas curriculares anclados en los métodos tradicionales que hacian que las instituciones tuvieran un carácter hegemónico.

Hoy en día al integrarse las tecnologías en los procesos de enseñanza - aprendizaje es necesario reconocer elementos institucionales, de formación y personales que intervienen en ello.
La adopción y el uso de las tecnologías no están determinados por la edad de los estudiantes y profesores, sino por una multitud de factores como: el acceso o la disposición, la utilidad y facilidad que advierten de estas, las valoraciones, las barreras percibidas, la formación para su uso, entre otros (Arancibia, M, Cabero, A y Valdivia, Z, 2019, pág. 106).

La incorporación de nuevas tecnologías portátiles digitales en el contexto educativo ha permitido la flexibilización, ubicuidad, a sincronía, conectividad e interactividad en la formación de los estudiantes, así como el surgimiento de nuevos modelos educativos, "ya que sus cualidades constitutivas ofrecen una serie de ventajas en el desarrollo de prácticas pedagógicas innovadoras que periten maximizar su alcance y capacidades" (Organista, S, López, O, y Reyes, R, 2020, pág. 37).

Las destrezas digitales, en cierta forma, dependen del aprovechamiento de las herramientas que los artefactos tecnológicos brindan y el uso frecuente de estos para ejecutar diversas actividades en contextos variados como la educación. Las Tecnologías de la Información y Comprensión - TIC - en "los sistemas educativos se enfocan en el apoyo a los procesos de enseñanza y aprendizaje, donde estudiantes y docentes adquieren capacidades en el uso eficaz de las tecnologías digitales para ser competentes en la sociedad moderna" (Laitón, E, Gómez, S, Sarmiento, R y Mejia, C, 2017, pág. 84).

Las TIC transformaron la manera de interacción entre las personas, revolucionando muchos campos de la actividad humana. Tal como lo describe Camacho (2009), "para esta sociedad resulta imperante que el colectivo se constituya en redes sociales dotadas de mecanismo de comunicación e interacción apoyados en las TIC" (pág. 5). Destacándose la educación, en donde, a presencia física ya no es una condición 
necesaria para enseñar y aprender, debido a que haciendo uso de las TIC se pueden alcanzar las metas propuestas en materia educativa.

Sin embargo, en estos escenarios el papel del docente es sumamente importante en la medida en que "desde las prácticas pedagógicas y los estilos de enseñanza de los docentes se debe promover la mediación y el intercambio entre los estudiantes" (Rendón, 2012, pág. 70), sin importar si se trata de espacios virtuales. Es por ello, que al tener claridad que el elemento fundamental de la educación es la información tal como lo afirman Martín, E y Solari, M (2011), la cual se debe enfocar en la persona competente, quien es la que puede movilizar ese conocimiento y utilizarlo para la toma de decisiones y resolver mejor las situaciones que se nos plantea en el proceso de enseñanza aprendizaje.

Desde este punto de vista tal como lo establecen Rivera (2011), Calderón (2012), y Velásquez (2012) la formación del docente para el uso de las TIC debe estar orientada a concebir los cambios que la tecnología trae de la mano, sin que ésta afecte su proceso de enseñanza; por el contrario, les va a servir para ampliar su visión frente a las competencias que habitualmente utiliza para su ejercicio profesional.

Así, "la integración de las tecnologías de la información y la comunicación en la educación se considera casi unánimemente, en la actualidad, como una de las oportunidades claves para mejorar e innovar la educación y el aprendizaje" (Pérez y Pi, 2013, pág. 5).

Esta integración ha permitido aumentar la eficiencia en el sistema educativo, a través del mejoramiento del rendimiento académico en lo que se refiere a la adquisición de las "competencias básicas y profesionales; mayor motivación de los estudiantes; mejora de acceso a contenidos actualizados; innovación de los escenarios y metodologías pedagógicas que en general dan una mejor adaptación del proceso de enseñanza en centro educativo" (Pérez y Pi, 2013, pág. 5).

Con lo abordado hasta ahora se ha hecho referencia a la virtualidad de la educación, permitiendo dar paso a las competencias docentes; para ello, se debe comprender que la "dimensión cognitiva de la competencia personal - profesional es precisa para que la función educativa tenga sentido. Dichas competencias hacen referencia a cuatro ámbitos: relación con los alumnos, compañeros y directivos, familias y comunidad y consigo mismo" (Gallego, M; Gámiz, V y Gutiérrez, E, 2010, pág. 2).

Lo referido da paso a las competencias digitales en la formación docente como parte del vértice, que se integra en el triángulo dado entre la formación - innovación e investigación.

Las competencias digitales se han asociado a dos objetivos clave de la preparación de los futuros docentes: por un lado, conocer y reflexionar sobre el contexto tecnológico en el que se desenvuelven sus alumnos $y$, por otro, desarrollar nuevas habilidades que les permitan utilizar las tecnologías para favorecer aprendizajes significativos. A la competencia cognitiva (sé) se añaden la competencia funciona (sé hacer), la competencia personal (sé estar) y la competencia ética (sé ser), referida esta última a los valores (Gallego, M; Gámiz, V y Gutiérrez, E, 2010, pág. 3).

Como se puede observar la competencia digital es vital para el presente y futuro del docente, y más aún si se tiene en cuenta la coyuntura actual con relación a la pandemia COVID - 19; de ahí la necesidad de que las competencias docentes y digitales se unan para lograr su incorporación en las actividades de enseñanza - aprendizaje. "La formación del profesorado no puede reducirse a la adquisición de competencias digitales o 
destrezas tecnológicas per se, sino que debe basarse en su aplicación didáctica" (Gallego, M; Gámiz, V y Gutiérrez, E, 2010, pág. 3).

Se percibe de lo expresado que las competencias integran un campo más amplio, desde el cual se pueden ejercer las actividades inherentes para el desarrollo de las competencias pedagógicas que permiten la trazabilidad de los conocimientos docentes de cara a la virtualidad de la educación.

Como complementó se debe tener en cuenta que, en la última década, las competencias se han constituido en el eje articulador del sistema educativo de Colombia. El Ministerio de Educación Nacional define competencia como el "conjunto de conocimiento, habilidades, actitudes, comprensiones y disposiciones cognitiva, socioafectivas y psicomotoras apropiadamente relacionadas entre sí para facilitar el desempeño flexible, eficaz $y$ con sentido de una actividad en contextos relativamente nuevos y retadores" (Ministerio de Educación Nacional, 2013, pág. 31).

De este modo, las competencias se encuentran en el momento de integración ya que incentiva el aprendizaje mediado por TIC en toda la comunidad educativa, desde cinco tópicos: competencia tecnológica, competencia comunicativa, competencia pedagógica, competencia de gestión y competencia investigativa. Las cuales hacen referencia a:

- Competencia tecnológica: El propósito de la integración de TIC en la educación ha sido mejorar los procesos de enseñanza - aprendizaje, así como la gestión escolar. Es "la capacidad para seleccionar y utilizar de forma pertinente, responsable y eficiente una variedad de herramientas tecnológicas entendiendo los principios que las rigen, la forma de combinarlas y las licencias que las amparan" (Ministerio de Educación Nacional, 2013, pág. 31).
- Competencia comunicativa: Las TIC facilitan la conexión entre "estudiantes, docentes, investigadores, y demás, incluso de manera anónima, y también permiten conectarse con datos, recursos, redes y experiencias de aprendizaje. Consiste en la capacidad para expresarse, establecer contacto y relacionarse en espacios virtuales y audiovisuales a través de diversos medios" (Ministerio de Educación Nacional, 2013, pág. 32).

- Competencia pedagógica: Es el saber propio de los docentes que se construyen en el momento que la comunidad investiga el sentido de lo que hace. "Las TIC han mediado algunas de las prácticas tradicionales y también han propiciado la consolidación de nuevas formas de aproximación al quehacer docente, enriqueciendo así el arte de enseñar" (Ministerio de Educación Nacional, 2013, pág. 32).

- Competencia de gestión: Se concentra en modular los factores asociados al proceso educativo, con el fin de imaginar de forma sistemática y sistémica lo que se quiere que suceda. "Para todos estos procesos existen sofisticadas tecnologías que pueden hacer más eficiente la gestión escolar. Es la capacidad de utilizar las TIC en la planeación, organización, administración y evaluación de manera efectiva de los procesos educativos" (Ministerio de Educación Nacional, 2013, pág. 33).

- Competencia investigativa: "EI eje alrededor del cual gira la competencia investigativa es la gestión de conocimiento y, en última instancia, la generación de nuevos conocimientos. Es la capacidad de utilizar las TIC para la transformación del saber y la generación de nuevos conocimientos" (Ministerio de Educación Nacional, 2013, pág. 33). 
Las TIC, como herramientas de gestión del conocimiento y facilitadoras de la comunicación global, juegan un papel importante en la adquisición de los saberes, ya que ayudan a mejorar las oportunidades de aprendizaje, facilitar el intercambio de información científica e incrementar el acceso a contenidos lingüista y culturalmente diversos, además de ayudar a promover la democracia, el diálogo y la participación cívica.

Por último, se ha hecho común las 17 competencias del docente moderno que constituyen un nuevo reto para los docentes en el rol que deben tener en los ambientes virtuales de formación, estas competencias son: compromiso, capacitación, organización y planificación, tolerancia, permitir preguntas, involucrar a los estudiantes, compartir historias, aprender y aplicar nuevas tecnologías, ser creativo, estimular a sus pupilos, dominar su inteligencia emocional, ser socialmente responsable, aprender un nuevo idioma, trabajo en equipo, comunicarse efectivamente, ser autocrítico y ser justo.

\section{LA VIRTUALIDAD DE LA EDUCACIÓN CON OCASIÓN DE LA PANDEMIA COVID - 19}

Como se expuso en el primer apartado la virtualidad de la educación ya tiene un recorrido amplio y reconocido en el ámbito educativo. Sin embargo, la virtualidad era una opción, para quienes no podían asistir al aula de clase, al método tradicional. Hoy por hoy la virtualidad dejó de ser voluntaria para convertirse en obligatoria, por lo menos, mientras el mundo vuelve a retomar su normalidad.

El sector educativo en cuestión de una semana se enfrentó al reto de garantizar la continuidad de su labor dentro de las nuevas condiciones. El cambio abrupto en marzo de 2020 llevó a la migración forzada tanto de estudiantes como del cuerpo docente y administrativos a la virtualidad
(Garavito, 2020, pág. 1).

Situación que puso a las instituciones educativas como el cuerpo docente, administrativo y directivas deberían estar preparados con la inmediatez que la urgencia puso al orden del día. Así, el actual modelo de educación que "se está desarrollando exigió la invención de nuevas formas de enseñar y aprender. Cada día surgen herramientas que enriquecen la manera de transmitir ideas, debatirlas, evaluarlas, editarlas, compararlas, compartirlas, etc., generando con ello una oportunidad para fortalecer las competencias docentes" (Peña, 2020, pág. 1).

Desde esta óptica se hacen presentes con más rigor la ética que deben tener los estudiantes al ser evaluados por sus docentes; la disciplina $y$ la automotivación, que es uno de los puntos más álgidos del actual modelo educativo; el respeto y la empatía, que debe ser promovido tanto por docentes como por los estudiantes; la curiosidad por el conocimiento, entre otros.

Todos estos conceptos responden también a la adaptación de las competencias de los docentes frente al cambio que tuvo la educación. "El reto de los sistemas educativo en los últimos meses ha sido mantener la virtualidad de la educación. Esto ha representado un desafío sin precedentes, pues los profesores tuvieron que generar sus propios aprendizajes para trabajar entornos virtuales" (Expósito, D y Marsollier, G, 2020, pág. 2).

Sin embargo, en Colombia al igual que en otros países de América Latina se ha logrado evidenciar que el sector de la educación no estaba preparado para la virtualidad en ninguna modalidad de formación, a lo que se suma las escasas competencias docentes en el ámbito virtual. Así lo manifestó la Unión Nacional de Estudiantes de Educación Superior - UNEES - señalando que "algunos profesores no están capacitados para hacer uso de las herramientas 
virtuales, haciendo aún más difícil el proceso de enseñanza - aprendizaje" (Baéz, 2020, pág. 1).

Con el cambio que trajo la tecnología nació la necesidad de que los docentes integraran a sus competencias, el perfil tecno - digital, lo que no era claro era que éstas tenían que integrarse de un día para otro, por el contexto presentado por la crisis sanitaria provocado por la pandemia de COVID - 19. La labor del docente tiene "que ser la de mentor, de guía para investigar y decidir los elementos necesarios, acompañando al alumnado en su interés y profundización individualmente según sus necesidades, ayudando a generar criterio. Una tarea más activa que pasiva, también en lo digital" (Cabrera, 2020, pág. 1).

Frente a esta situación los docentes deben trabajar más en la formación de sus competencias digitales, la forma de enseñar y evaluar; sin embargo, hay quienes consideran que esto es momentáneo y por ende no buscan cambiar mucho su forma de enseñar, perdiendo una gran oportunidad de adaptación que la pandemia ha traído.

Para que lo anterior se de y especialmente para que se pueda continuar en un proceso de ascenso hacia la calidad de la educación, desde la virtualidad se deben integrar ciertos aspectos, como:

Contar con los recursos tecnológicos adecuados y el servicio necesario para acceder al programa educativo; que la estructura y el contenido del curso virtual ofrezca un valor formativo; que se realicen aprendizajes efectivos y que sea un ambiente satisfactorio tanto para los estudiantes como para los profesores (Expósito, D y Marsollier, G, 2020, pág. 2).

Desde esta perspectiva se ha hecho visible un entusiasmo que responde a las exigencias actuales de la virtualidad, pero que se debe acompasar con lo señalado; pues de nada sirve que los docentes afiancen sus esfuerzos para alinear sus competencias con la virtualidad si no existen los recursos tecnológicos adecuados.

Llama la atención frente a las reflexiones planteadas, que también la pandemia ha ofrecido cambios positivos, en cuanto a la "posibilidad de aprender de otra manera, de un modo más autónomo, como estudiantes cada vez más responsables de su propio aprendizaje" (Baptista, P, Almazán, Z, Loeza, A, López, A y Cárdenas, D, 2020, pág. 43). Es por ello, que se deben afianzar los espacios para que la educación realmente cumpla con su misión, la educar con calidad, y no caer en lo que Feito (2020), Rogero (2020) y Morgan (2020) han analizado, al expresar que la educación está enfrentando una situación que dista mucho de contar con los elementos necesarios para la formación y que ahondan problemas ya reconocidos por el método tradicional de enseñanza: la cobertura y la calidad.

Lo referido es fundamentado por Ceresuela (2020) al expresar que la escuela ofrece muchas alternativas, además del aprendizaje; es el sitio donde se afianzan las bases para un buen ciudadano, es el lugar de juegos de interrelación donde los niños y jóvenes alternan sus vivencias y situaciones, también ayuda a delinear la personalidad e identidad que se va a ver reflejada en la educación superior; al respecto Cotino (2020) expresa que ante el COVID - 19 la tecnología no sustituyen el contacto que ofrece el método presencial de formación entre docentes y estudiantes, no sustituyen las relaciones interpersonales que forjan amistades y experiencias que la virtualidad no brinda.

Pero como dirían muchos es jlo que hay!, y queramos o no tenemos que adaptarnos, porque de lo contrario estaríamos destinados a vivir en el ostracismo, sin oportunidad alguna. Adicional, hay que aceptar que esta nueva 
visión del mundo permite que el rol del docente se transforme, al igual que el estudiante. Cornock (2020) indica que la responsabilidad ya no recae solamente en el docente, pues él es un mediador, permitiéndole al estudiante un mayor protagonismo debido a que éste tiene la responsabilidad de aportar contenidos y participar activamente en su aprendizaje. Entonces, como expresa Britez (2020) se podría comenzar a hablar que las competencias de los estudiantes también han cambiado al tener un rol más protagónico.

Las reflexiones hasta ahora planteadas llevan a establecer el avance que Colombia ha tenido frente a las exigencias del COVID - 19 en el entorno educativo. Así, el Ministerio de Educación Nacional (2020) fija los parámetros para enfrentar la pandemia, a través de unos lineamientos que buscan ofrecer la ruta a seguir por parte de las instituciones educativas. El Ministerio de Educación considera necesario "definir las estrategias pedagógicas que articulen las áreas, prioricen las competencias docentes, incluyan o fortalezcan las demandas actuales en pro del desarrollo integral y la experiencia de aprendizaje significativo" (Ministerio de Educación Nacional, 2020, pág. 49).

Esto con el fin de afianzar el proceso de alternancia entre el hogar y la institución educativa. Frente a esta realidad, no se puede dejar de lado que el mundo ha avanzado a la "llamada cuarta revolución industrial y que el COVID - 19 evidenció que la educación en Colombia, especialmente la pública, presenta enormes rezagos en apropiar herramientas tecnológicas para favorecer el aprendizaje de los estudiantes" (Abadía, 2020, pág. 1).

Este fenómeno pone en evidencia las inequidades que existen entre el sector público y privado en cuanto al acceso de la tecnología que puedan tener los estudiantes y que decir de la situación de los docentes que en la misma situación se ven afectados.

\section{CONCLUSIONES}

Los cambios que el docente ha tenido que hacer en su forma de enseñar, según las exigencias de la virtualidad, al tener que fusionar lo físico con lo digital, ha permitido que logré que sus competencias docentes generen un equilibrio en el entorno de enseñanza - aprendizaje.

La pandemia COVID - 19 ha exigido en los docentes un capacidad de adaptación e innovación en sus prácticas y particularmente en la forma de enseñar que le exige día a día reinventarse y reconocer las diferentes herramientas que la tecnología ofrece y que no merece ser excluida; también ha puesto de manifiesto los atrasos y temores de los docentes frente a la situación, generando en quienes no desean asumir los cambios un rechazo y por ende una pérdida de trabajo, de estabilidad emocional e inclusive cuadros de depresión.

Desde este escenario es importante promover los espacios para que los docentes afiancen sus competencias digitales y así continuar desempeñando un papel estelar en la formación de los estudiantes; para ello, deben comprender que las competencias no son estáticas, que el mundo de hoy cambia al instante, que el no estar preparándose lo va a llevar a perder las posibilidades labores e inclusive sociales, pues la realidad es que el mundo día a día se virtualiza más.

La pandemia también ha evidenciado las desigualdades educativas, y no necesariamente frente a la matrícula, sino con relación al acceso a la tecnología por parte de los docentes para incursionar y aprender sobre el mundo digital; se puede decir que el docente se ha quedado "solo" frente a la pantalla del computador cuando lo tienen, o ante la necesidad de conseguir los elementos para poder desarrollar su trabajo; esto se agudiza más en las zonas rurales donde la cobertura tecnológica es muy deficiente y los 
docentes no cuentan con procesos de formación que les ayuden a reconocer el ámbito virtual.

Se concluye que el sistema educativo colombiano no está realmente adecuado para la educación virtual, que también se encuentra en un proceso de readaptación y de modernización para ofrecer la infraestructura y los lineamientos normativos para que las instituciones tengan una ruta a seguir en este nuevo modelo de educación.

Existe una clara necesidad de adaptar la infraestructura, el recurso humano, los procesos logísticos y todo aquello que intervenga de forma directa o indirecta en el proceso educativo para lograr enfrentar lo que el futuro trae; pues como muchos opinan este es apenas el comienzo de muchas pandemias y el reacomodamiento de la economía y de los escenarios sociales, educativos, laborales, entre otros.

\section{REFERENCIAS BIBLIOGRÁFICAS}

Abadía, A. (30 de marzo de 2020). El reto que el sector educativo en Colombia debe superar tras la pandemia. Obtenido de https://www.javeriana.edu.co/ documents/12789/11569759/P\%C3\%A 1g.+9.+El+reto+del+sector+educativo. +HJ+marzo+2020+web.pdf/bf0db075be31-413f-8496-e6d72ec72bf9

Arancibia, M, Cabero, A y Valdivia, Z. (2019). Estudio comparativo entre docentes y estudiantes sobre aceptación y uso de tecnologías con fines educativos en el contexto chileno. Apertura, 11(1), 104-119. doi:https://doi.org/10.32870/ Ap.v11n1.1440

Arraiz, G. (abril - septiembre de 2012). La virtualidad: un escenerio posible para la construcción de conocimientos matemáticosA. Apertura, 4(1), 76-85.

Baéz, J. (16 de marzo de 2020). La incapacidad de las Instituciones de Educación Superior (IES). Obtenido de https:// www.contagioradio.com/eduacionvirtual-por-covid-19-una-decision-parala-que-no-estamos-preparados/

Baptista, P, Almazán, Z, Loeza, A, López, A y Cárdenas, D. (31 de julio de 2020). Encuesta Nacional a docentes ante el COVID - 19. Retos para la educación a distancia. Revista Lationamericana de Estudios Educativos, L(1), 41 - 88. doi:https://doi.org/10.48102/ rlee.2020.50.ESPECIAL.96

Britez, M. (13 de mayo de 2020). La educación ante el avance del COVID - 19 en Paraguay. Comparativo con países de Triple Frontera. Scielo, 2(1), 1-15. doi:https://doi.org/10.1590/ SciELOPreprints.22

Cabrera, M. (1 de junio de 2020). COVID - 19 y formación virtual: cómo adoptar competencias digitales en cuestión de días. Obtenido de https:// theconversation.com/covid-19-yformacion-virtual-como-adoptarcompetencias-digitales-en-cuestion-dedias-139698

Calderón, N. (13 de Diciembre de 2012). Los Valores a Partir de las Tic. Obtenido de Slideshare.net: http://es.slideshare. net/natalia64/valores-a-partir-de-las-ticproyecto-de-luzmila

Camacho, I. (2009). Educación en valores en ambientes virtuales. Etic@ net, VII(8), $1-31$.

Ceresuela, P. (25 de abril de 2020). ¿Perder el año escolar? 4 expertos responden. Obtenido de https://www.latercera. com/nacional/noticia/perder-elano-escolar-4-expertos-responden/ OB6PAPUKCZB35IF62KVRCVDU44/ 
Cornock, M. (12 de march de 2020). caling up online learning during the coronavirus. (Covid-19) pandemic. Obtenido de https://mattcornock.co.uk/technologyenhanced-learning/scaling-up-onlinelearning-during-the-coronavirus-covid19-pandemic/

Cotino, H. (2020). La enseñanza digital en serio y la educación en tiempos del coronavirus. Revista de Educación y Derecho, 21-29. doi:https://doi. org/10.1344/REYD2020.21.31283

Expósito, D y Marsollier, G. (julio - diciembre de 2020). Virtualidad y educación en tiempos de COVID - 19. Un estudio empírico en Argentina. Educación y Humanismo, 22(39), 1-22. doi:https:// doi.org//10.17081/eduhum.22.39.4214

Feito, R. (2020). Este es el fin de la escuela tal y como la conocemos. Unas reflexiones en tiempo de confinamiento. Revista Sociología de la Educción, 13(2), 156 - 163. doi:https://doi. org/10.1177/0031721720923799

Gallego, M; Gámiz, V y Gutiérrez, E. (diciembre de 2010). El futuro docente ante las competenciasenelusodelastecnologías de la información y comunicación para enseñar. EDUTEC(34), 1-18. doi: https:// doi.org/10.21556/edutec.2010.34.418

Garavito, C. (1 de junio de 2020). Los 'pro' de la evolución forzada hacia la virtualidad en la educación. Obtenido de Colegio de Estudios Superiores de Administración: https://www.cesa.edu.co/news/lospro-de-la-evolucion-forzada-hacia-lavirtualidad-en-la-educacion/

Laitón, E, Gómez, S, Sarmiento, R y Mejia, C. (2017). Competencia de prácticas inclusivas: las TIC y la educación inclusiva en el desarrollo profesional docente. Sophia, 13(2), 81-95. doi:http:// dx.doi.org/ 10.18634/sophiaj.13v.2i.502

Martín, E y Solari, M. (2011). ¿Puede el enfoque de las competencias contribuir a la inclusión y la ciudadanía? Tejuelo. Didáctica de la lengua y la literatura. (12), 9-25.

Medina, G. (2019). La virtualidad de la educación, un reto en el aprendizaje universitario. Revista Iberoamericana de Educación Superior, X(29), 215$217 . \quad$ doi:https://doi.org/10.22201/ iisue.20072872e.2019.29.532

Ministerio de Educación Nacional. (2013). Competencias TIC para el desarrollo profesional docente. Bogotá, D.C: Mineducación.

Ministerio de Educación Nacional. (2020). Lineamientos para la prestación del servicio de educación en casa y en presencialidad bajo el esquema de alternancia y la implementación de prácticas de bioseguridad en la comunidad educativa. Bogotá, D.C.: MEN.

Morgan, H. (28 de april de 2020). Best Practices for Implementing Remote Learning during a Pandemic. A Journal of Educational, 93, 135-141. doi:https:// doi.org/10.1080/00098655.2020.17514 80

Nieto, G. (julio-diciembre de 2012). Educación virtual o virtualidad de la educación. Revista historia educativa latinoamericana, 14(19), 137150. doi:http://dx.doi.org/10.9757/ Rhela.19.06 
Organista, S, López, O, y Reyes, R. (2020). Elaboración de módulos audiovisuales para mejorar las habilidades digitales de estudiantes universitarios. Apertura, 12(2), 36-51. doi:http://dx.doi. org/10.32870/Ap.v12n2.1893

Peña, F. (30 de julio de 2020). Nuevo modelo de educación para el futuro. Obtenido de https://www.cesa.edu.co/news/nuevomodelo-de-educacion-para-el-futuro/

Pérez y Pi. (2013). La integración de las TIC y los libros digitales en la educación. Barcelona: Planeta.

Rendón, U. (2012). Las convivencias y las competencias ciudadanas en la universidad. Uni-pluri/versidad, 12(2), 57-72.

Rivera, S. (2011). Formación del docente para el usodelasTIC.Obtenidodehttp://gte2.uib. es/edutec/sites/default/files/congresos/ edutec11/Ponencias/Mesa\%201-anx/ Formaci \% C 3 \% B 3 n \% 20 del \% 20 docente $\% 20$ para $\% 20$ el\%20uso $\% 20$ de\%20las\%20TIC.pdf

Rogero, G. (2020). La ficción de educar a distancia. Revista de Sociología de la Educación, 13(2), 174-182. doi:http:// dx.doi.org/10.7203/RASE.13.2.17126.

Velásquez, A. (2012). Una mirada crítica a la didáctica de las ciencias, la educación científica y el papel de las TIC en la educación superior. (Tesis de grado). Ibagué: Universidad del Tolima. 